\title{
Characteristics of a tunneling quantum-dot infrared photodetector operating at room temperature
}

\author{
P. Bhattacharya, ${ }^{\text {a) }}$ X. H. Su, and S. Chakrabarti \\ Solid State Electronics Laboratory, Department of Electrical Engineering and Computer Science, \\ University of Michigan, Ann Arbor, Michigan 48109-2122 \\ G. Ariyawansa and A. G. U. Perera \\ Department of Physics and Astronomy, Georgia State University, Atlanta, Georgia 30303
}

(Received 13 December 2004; accepted 21 March 2005; published online 3 May 2005)

\begin{abstract}
We report high-temperature $(240-300 \mathrm{~K})$ operation of a tunneling quantum-dot infrared photodetector. The device displays two-color characteristics with photoresponse peaks at $\sim 6 \mu \mathrm{m}$ and $17 \mu \mathrm{m}$. The extremely low dark current density of $1.55 \mathrm{~A} / \mathrm{cm}^{2}$ at $300 \mathrm{~K}$ for $1 \mathrm{~V}$ bias is made possible by the tunnel filter. For the $17 \mu \mathrm{m}$ absorption, the measured peak responsivity is $0.16 \mathrm{~A} / \mathrm{W}$ $(300 \mathrm{~K})$ for a bias of $2 \mathrm{~V}$ and the specific detectivity $D^{*}$ is $1.5 \times 10^{7} \mathrm{~cm} \mathrm{~Hz}^{1 / 2} / \mathrm{W}(280 \mathrm{~K})$ for a bias of $1 \mathrm{~V}$. Excellent performance characteristics are also measured for the $6 \mu \mathrm{m}$ photoresponse. (c) 2005 American Institute of Physics. [DOI: 10.1063/1.1923766]
\end{abstract}

A significant reduction in the cost of an infrared camera is possible if the traditional cooling systems with liquid nitrogen are replaced by thermoelectric coolers, or the array can be operated at room temperature. There is, therefore, an urgency to develop infrared photodetectors that operate at elevated temperatures. The quantum-dot infrared photodetector (QDIP) ${ }^{1-3}$ has emerged as an interesting, and potentially viable device, wherein three-dimentional quantum confinement promises low dark currents, leading to a large detectivity. Additionally, in these devices, polarization selection rules allow absorption of normally incident light and the long effective carrier lifetime $\sim 100$ 's of picoseconds, confirmed by theory ${ }^{4}$ and experiments, ${ }^{5}$ provides the potential for large responsivity. Rapid progress has been made in the development of these devices and recently reported performance characteristics $^{6-11}$ (low dark currents, peak responsivity $\geqslant 0.5 \mathrm{~A} / \mathrm{W}$ and specific detectivity $\sim 10^{10}-10^{11} \mathrm{~cm} \mathrm{~Hz}^{1 / 2} / \mathrm{W}$ in the temperature range $\sim 80-150 \mathrm{~K}$ ) are cause for optimism.

Unlike those in bulk $p-n$ junction detectors or in quantum-well infrared photodetectors, the density of states of the self-organized $\operatorname{In}(\mathrm{Ga}) \mathrm{As} / \mathrm{GaAs}$ quantum dots in QDIPs is represented by broadened $\delta$-functions for the electron and hole ground and excited states. Even though the density of states is discrete for low-energy states, it tends to become like three-dimensional continuous states at high energies. As a result, at temperatures above $\sim 150 \mathrm{~K}$, the electron occupation is dominated by the excited states. Therefore, in the conventional QDIP, dark current benefits are difficult to sustain at temperatures above $150 \mathrm{~K}$. The reduction of dark current at high temperatures of operation is one of the biggest challenges in the design of QDIPs. The problem stems from the commonality of the transport paths of carriers contributing to the dark- and photocurrent. Hence, any heterostructure design aimed at reducing the dark current also reduces the photocurrent and responsivity. We have recently reported the properties of a device-the tunneling QDIP (Ref. 12) - in which the electrons contributing to the photocurrent are selectively collected from the quantum dots

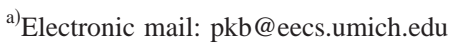

by resonant tunneling, while these same tunnel barriers block the electrons, with a broad energy distribution, that contribute to the dark current. We have shown theoretically and experimentally, a reduction of dark current by two orders of magnitude in tunneling QDIPs. ${ }^{12}$ In this letter, we demonstrate room temperature operation of tunneling QDIPs, with a slightly modified design, with response peaks at wavelengths of $\sim 6 \mu \mathrm{m}$ and $17 \mu \mathrm{m}$.

The band diagram of the tunneling QDIP (T-QDIP) under an applied transverse bias is shown in Fig. 1(a). The double-barrier resonant tunneling heterostructure serves to decouple the dark- and photocurrent. The purpose of the single $\mathrm{Al}_{0.1} \mathrm{Ga}_{0.9} \mathrm{As}$ barrier, asymmetrically placed on the side of the dot opposite to the tunnel barriers, is to create well-defined quasi-bound final states in the quantum well for the photoexcited electrons. These states are also made to resonate with the tunneling states in the $\mathrm{In}_{0.1} \mathrm{Ga}_{0.9} \mathrm{As} / \mathrm{Al}_{0.3} \mathrm{Ga}_{0.7} \mathrm{As}$ double barrier heterostructure. The tunnel probability is therefore near unity, as confirmed by calculations, for photoexcited electron transitions whose energies coincide with the QD bound state-to-well final state energy difference. The incorporation of the $\mathrm{Al}_{0.1} \mathrm{Ga}_{0.9} \mathrm{As}$ barrier also allows slight tuning of the peak response wavelength without having to change the quantum dot heterostructure design or the growth parameters. By simply adjusting the separation of the $\mathrm{Al}_{0.1} \mathrm{Ga}_{0.9} \mathrm{As}$ barrier from the quantum dot, the energy position of the quasi-bound state in the well can be tuned. The bound state energies in the quantum dot, as indicated in Fig. 1(a), were calculated by an eight-band $\mathbf{k} \cdot \mathbf{p}$ model $^{13}$ and the energy level in the quantum well is calculated by solving the one-dimensional Schrödinger equation including the presence of the wetting layer. The complete device heterostructure, grown by molecular-beam epitaxy, is schematically illustrated in Fig. 1(b). The GaAs and AlGaAs layers were grown at $610^{\circ} \mathrm{C}$ and the quantum dot layers were grown at $500{ }^{\circ} \mathrm{C}$. Mesashaped vertical devices for top illumination were fabricated by standard photolithography, wet chemical etching, and contact metallization techniques. The $n$-type top ring contact and the bottom contact were formed by evaporated $\mathrm{Ni} / \mathrm{Ge} / \mathrm{Au} / \mathrm{Ti} / \mathrm{Au}=250 / 325 / 650 / 200 / 2000 \AA$. The radius 


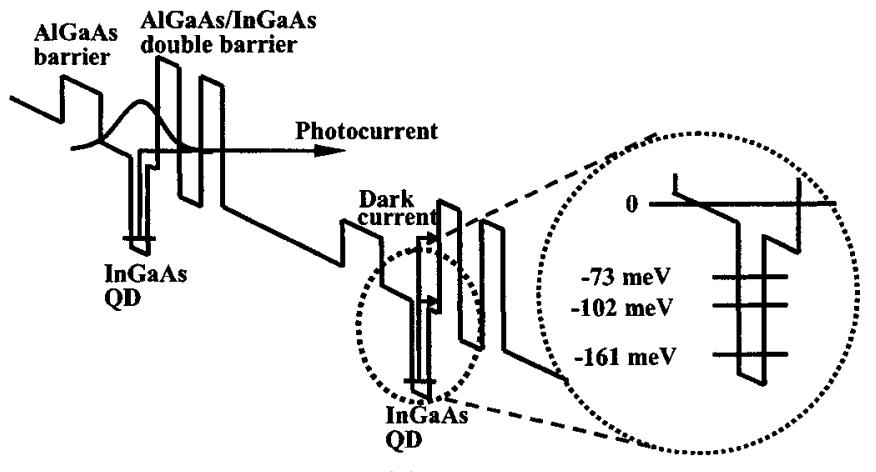

(a)

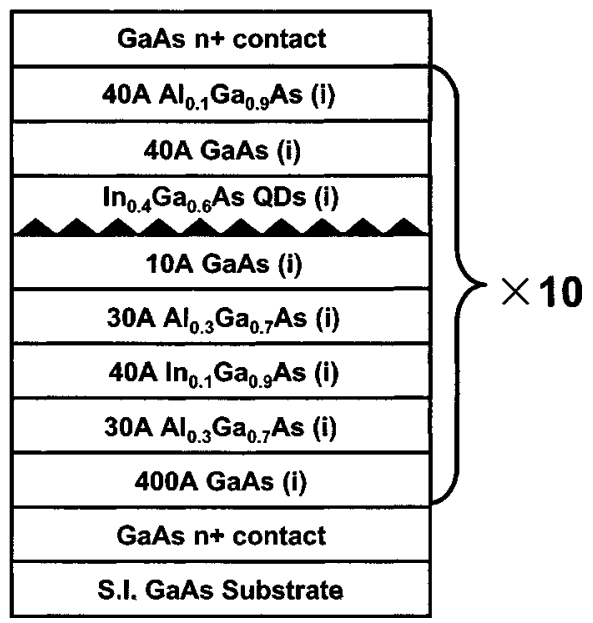

(b)

FIG. 1. (a) Schematic diagram of the conduction band profile of the T-QDIP under an applied transverse bias along with the calculated bound state energies in the dots and wells. (b) Schematic heterostructure of a T-QDIP grown by molecular-beam epitaxy.

of the illuminated area varies from 100-300 $\mu \mathrm{m}$. For testing purpose, devices are mounted onto chip carriers with silver epoxy and then bonded to the carrier leads using gold wire. The chip carrier is inserted into a liquid-helium cryostat for low-temperature measurements.

While the devices have been characterized in all the wavelength ranges of interest, in this letter, we mainly focus on their long wavelength detection characteristics at high temperatures. The dark current-voltage characteristics in the temperature range $240-300 \mathrm{~K}$, measured with a HP 4145 Semiconductor Parameter Analyzer, are shown in Fig. 2. The dark currents are substantially lower than those measured in

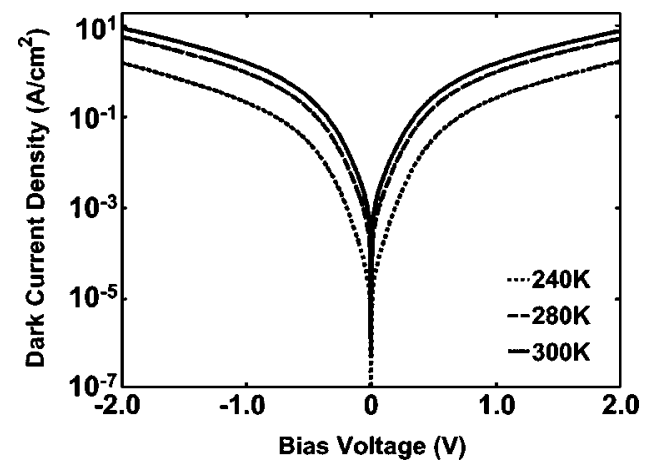

FIG. 2. Measured dark current density as a function of bias in the temperature range $240-300 \mathrm{~K}$.
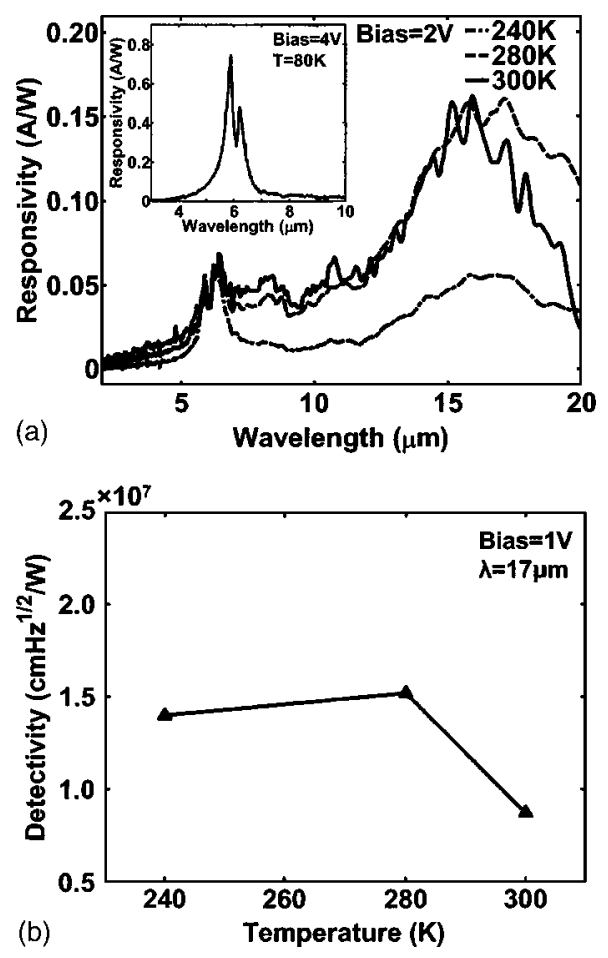

FIG. 3. (a) Measured spectral responsivity of T-QDIP in the temperature range $240-300 \mathrm{~K}$ under $2 \mathrm{~V}$ bias. The inset shows spectral responsivity at $80 \mathrm{~K}$ under a bias of $4 \mathrm{~V}$. (b) Peak detectivity as a function of temperature for the $17 \mu \mathrm{m}$ response derived from noise spectra measurements.

identical QDIPs without the tunneling double barriers. As stated earlier, we have reported ${ }^{12}$ a measured and calculated reduction by nearly two orders of magnitude. From the data of Fig. 2, the values of $J_{\text {dark }}$ at a bias of $1 \mathrm{~V}$ are $0.21,0.96$, and $1.55 \mathrm{~A} / \mathrm{cm}^{2}$ at 240,280 , and $300 \mathrm{~K}$, respectively.

The spectral response and calibrated responsivity, under normal incidence, were measured with a globar broadband source $(1-20 \mu \mathrm{m})$ and a BOMEM MB-155 Fourier transform infrared spectrophotometer. The spectral response of the QDIP under test and a composite bolometer with a known sensitivity are concurrently measured with the same combination of optical elements. Figure 3(a) shows the spectral response measured with an applied bias of $2 \mathrm{~V}$ in the temperature range $240-300 \mathrm{~K}$. There are two distinct absorption peaks, centered $\sim 6 \mu \mathrm{m}$ and $17 \mu \mathrm{m}$ and a weak response around $11 \mu \mathrm{m}$. On closer examination of the $6 \mu \mathrm{m}$ transition, shown in the inset for measurement at $80 \mathrm{~K}$ with $4 \mathrm{~V}$ bias, the spectral response exhibits closely spaced dual peaks with an energy separation of $\sim 15 \mathrm{meV}$. The dual peaks arise from coupling and splitting of the wave functions of the quantum-well states and the bound states of the double-barrier heterostructure and provide evidence of resonant tunneling in the operation of the device. The measured energy separation between the peaks agrees well with the calculated valued of $13.5 \mathrm{meV}$. Careful examination of the background absorption indicates that the dip between the peaks is not due to water and $\mathrm{CO}_{2}$ absorption. By changing the bias, small redshifts have been observed for both the dual peaks and the dip between them. It may be noted that the peak responsivity is $\sim 0.75 \mathrm{~A} / \mathrm{W}$ and the quantum efficiency is $16 \%$ for the $6 \mu \mathrm{m}$ response at a bias of $4 \mathrm{~V}$.

It is worthwhile to note that the long-wavelength $(17 \mu \mathrm{m})$ absorption becomes significant at temperatures higher than $200 \mathrm{~K}$ and, as is evident from Fig. 3(a), its peak 
responsivity, $\sim 0.16 \mathrm{~A} / \mathrm{W}$, at $300 \mathrm{~K}$ is even higher than that of the $6 \mu \mathrm{m}$ peak. With reference to the calculated energies of the bound and quasi-bound states in the dot and well, shown in Fig. 1(a), it is apparent that the absorption peak at $\sim 6 \mu \mathrm{m}$ results from transition of photoexcited electrons from the dot ground state to the quasi-bound state in the well $(\Delta E=161 \mathrm{meV})$. The broad peak centered at $17 \mu \mathrm{m}$ results from transitions from the second-excited state of the dot to the well state $(\Delta E=73 \mathrm{meV})$. The width of the peak is due to the transition being in the long-wavelength infrared region. In fact, the transition linewidth is only $\sim 26 \mathrm{meV}$, which is remarkably close to the inhomogeneous broadening of the QD states at $300 \mathrm{~K}$. We believe that the $17 \mu \mathrm{m}$ transition is dominant at high temperatures because the probability of occupation of the dot excited states increases with temperature. Due to the symmetry of the dot geometry, the excited states have a higher degeneracy than the ground state. The degeneracy of the second excited states is $8,{ }^{13}$ while it is 2 for the ground state. Therefore, the total number of carriers in the excited states can be comparable to, or larger, than that in the ground state at high temperatures although the occupation probability is lower. Additionally, for the same incident power, there are more photons at $17 \mu \mathrm{m}$ than at $6 \mu \mathrm{m}$. These characteristics help to explain a higher absorption peak at $17 \mu \mathrm{m}$ compared to that at $6 \mu \mathrm{m}$ for temperatures of 280 and $300 \mathrm{~K}$. It may be noted that the weak transition at $\sim 11 \mu \mathrm{m}$ corresponds almost exactly to the energy separation between the first-excited dot state and the well state $(\Delta E$ $=102 \mathrm{meV}$ ). The selection rules may account for the weak transition. The wave function of the first-excited dot states is antisymmetrical in space and the wave function of the final states in the well is almost symmetrical near the dot layer. These give rise to a small transition momentum matrix as well as low responsivity. In contrast, all initial and final states corresponding to the $6 \mu \mathrm{m}$ and $17 \mu \mathrm{m}$ transitions are symmetrical and the transitions are allowed. Finally, it is extremely important to note that it is the low dark current in the T-QDIP that makes detection possible at these high temperatures.

The QDIP photocurrent signal and noise are measured with a Keithley 428 Current Sensitive Amplifier and an Ono Sokki CF-360F fast Fourier transform (FFT) Analyzer, which displays an FFT spectrum of voltage versus frequency. A thick copper plate was used as a radiation block to provide the dark conditions for the measurement. The specific detectivity $D^{*}$, which is a measure of the signal-to-noise ratio of the device, was calculated from the noise density spectra and the peak responsivity. The values of $D^{*}$ for the $17 \mu \mathrm{m}$ response at a bias of $1 \mathrm{~V}$ are plotted in Fig. 3(b) as a function of temperature. The values of $D^{*}$, in the $10^{7} \mathrm{~cm} \mathrm{~Hz}^{1 / 2} / \mathrm{W}$ range, are reasonable at these high temperature ${ }^{14}$ and we are confident that with some redesigning of the device heterostructure, $D^{*} \sim 10^{9} \mathrm{~cm} \mathrm{~Hz}^{1 / 2} / \mathrm{W}$ can be demonstrated at room temperature. The measured $D^{*}$ for the $6 \mu \mathrm{m}$ absorption peak is $2.4 \times 10^{10} \mathrm{~cm} \mathrm{~Hz}^{1 / 2} / \mathrm{W}$ for a bias of $2 \mathrm{~V}$ at $80 \mathrm{~K}$.

In conclusion, we report the characteristics of a twocolor tunneling QDIP at high temperatures. $300 \mathrm{~K}$ operation is made possible by the incorporation of a double-barrier tunnel filter, which reduced the dark current to very low levels.

This work is supported by the Army Research Office (MURI program) under Grant No. DAAD19-01-1-0462 and at Georgia State University by the National Science Foundation under Grant No. ECS-0140434.

${ }^{1}$ J. Phillips, K. Kamath, and P. Bhattacharya, Appl. Phys. Lett. 72, 2020 (1998).

${ }^{2}$ S. Maimon, E. Finkman, and G. Bahir, Appl. Phys. Lett. 73, 2003 (1998).

${ }^{3}$ D. Pan, E. Towe, and S. Kennerly, Appl. Phys. Lett. 73, 1937 (1998).

${ }^{4}$ B. Kochman, A. D. Stiff-Roberts, S. Chakrabarti, J. D. Phillips, S. Krishna, J. Singh, and P. Bhattacharya, IEEE J. Quantum Electron. 39, 459 (2003).

${ }^{5}$ J. Urayama, T. B. Norris, J. Singh, and P. Bhattacharya, Phys. Rev. Lett. 86, 4930 (2001).

${ }^{6}$ E. Kim, A. Madhukar, Z. Ye, and J. C. Campbell, Appl. Phys. Lett. 84, 3277 (2004).

${ }^{7}$ S. Krishna, S. Raghavan, G. von Winckel, A. Stintz, G. Ariyawansa, S. G. Matsik, and A. G. U. Perera, Appl. Phys. Lett. 83, 2745 (2003).

${ }^{8}$ S. Raghavan, P. Rotella, A. Stintz, B. Fuchs, S. Krishna, C. Morath, D. A. Cardimona, and S. W. Kennerly, Appl. Phys. Lett. 81, 1369 (2002).

${ }^{9}$ L. Jiang, S. S. Li, N. T. Yeh, J. I. Chie, C. E. Ross, and K. S. Jones, Appl. Phys. Lett. 82, 1986 (2003).

${ }^{10}$ S. Chakrabarti, A. D. Stiff-Roberts, P. Bhattacharya, S. D. Gunapala, S. Bandara, S. B. Rafol, and S. W. Kennerly, IEEE Photonics Technol. Lett. 16, 1361 (2004).

${ }^{11}$ S. Chakrabarti, A. D. Stiff-Roberts, P. Bhattacharya, and S. W. Kennerly, Electron. Lett. 40, 197 (2004).

${ }^{12}$ X. H. Su, S. Chakrabarti, A. D. Stiff-Roberts, J. Singh, and P. Bhattacharya, Electron. Lett. 40, 1082 (2004).

${ }^{13}$ H. Jiang and J. Singh, Phys. Rev. B 56, 4696 (1998).

${ }^{14}$ G. M. Williams and R. E. DeWames, J. Electron. Mater. 24, 1239 (1995). 